\title{
Evaluation of the Effect of Probiotic Bacillus coagulans Unique IS2 on Mutans Streptococci and Lactobacilli Levels in Saliva and Plaque: A Double-Blind, Randomized, Placebo-Controlled Study in Children
}

\author{
M. Ratna Sudha $\mathbb{D}^{1},{ }^{1}$ Jayanthi Neelamraju ${ }^{D},{ }^{1}$ M. Surendra Reddy, ${ }^{2}$ and Manoj Kumar ${ }^{2}$ \\ ${ }^{1}$ Centre for Research and Development, Unique Biotech Ltd., Plot No. 2, Phase-II, Alexandria Knowledge Park, Hyderabad, \\ Telangana 500 078, India \\ ${ }^{2}$ Department of Pedodontics and Preventive Dentistry, SVS Institute of Dental Sciences, Mahbubnagar, Telangana, India
}

Correspondence should be addressed to M. Ratna Sudha; sudha@uniquebiotech.com

Received 19 June 2020; Revised 9 December 2020; Accepted 18 December 2020; Published 30 December 2020

Academic Editor: Andrea Scribante

Copyright (C) 2020 M. Ratna Sudha et al. This is an open access article distributed under the Creative Commons Attribution License, which permits unrestricted use, distribution, and reproduction in any medium, provided the original work is properly cited.

\begin{abstract}
Background. Probiotic organisms are known to reduce caries causing microorganisms mutans streptococci and lactobacilli. Aim of the Study. To evaluate the effect of probiotic Bacillus coagulans Unique IS2 on mutans streptococci and lactobacilli levels in saliva and plaque in children. Introduction. Dental caries or tooth decay is because of the demineralization of the tooth enamel leading to the breakdown of the enamel causing cavities to be formed. Demineralization of the tooth happens because of the acid secreted by bacteria like mutans streptococci and lactobacilli. It is now suggested that probiotic usage prevents the overgrowth of these pathogenic microbes, thereby reducing caries activity. Methodology. In this double-blind, randomized, placebo-controlled study, 48 children with ages ranging from 5 to 15 years were divided into two groups, the probiotic and placebo groups. Chewable tablets with and without probiotic Bacillus coagulans Unique IS2 were administered for two weeks. Stimulated saliva samples and plaque were collected at baseline and at the end of 14 days to measure the $\mathrm{pH}$, mutans streptococci, and lactobacilli count of saliva and plaque using chairside kits. Results. A statistically significant reduction in mutans streptococci and lactobacilli counts of both saliva and plaque samples was observed in the B. coagulans Unique IS2 treated group after 14 days of administration compared to the baseline and placebo group (using paired $t$-test). Conclusion. Probiotic Bacillus coagulans Unique IS2 (2 billion cfu) chewable tablet is effective in reduction and inhibition of caries causing mutans streptococci and lactobacilli levels in saliva and plaque in children.
\end{abstract}

\section{Introduction}

Oral diseases are a major health burden globally and people of all ages throughout their lifetime are affected [1]. Some of the common diseases that affect oral health are tooth decay (cavities), gingivitis, gum (periodontal) disease, halitosis (bad breath), and oral cancer.

It is estimated that oral diseases affect close to 3.5 billion people worldwide, with dental caries (tooth decay) being the most common condition [2]. It is estimated that 2.3 billion people globally suffer from caries of permanent teeth and more than 530 million children suffer from caries of primary teeth [3]. The percentage of school children affected by dental caries is $60 \%-90 \%$ and this is common for both developed and developing countries [4]. Some of the causes for dental caries include consumption of foods and beverages high in sugars, lack of oral hygiene, and absence of yearly dental check-ups. In developing countries where there is a lack of basic health facilities, oral health is grossly neglected leading to the high prevalence of dental caries especially in children [5]. Dental caries is a cause of pain, discomfort with enhanced sensitivity to cold and hot beverages apart from the loss of aesthetical appearance. The cost of dental treatments is high and hence prevention of dental 
caries by saving the teeth from decay is highly recommended instead of treatments aimed at drilling out the decay and putting in a filling (restoration) made from various materials [6]. Various methods to prevent dental caries have been employed [7] and these include treatment with fluoride (water fluoridation and fluoride toothpaste) and removal of dental plaque by use of antibacterial mouth rinses. Other methods to prevent dental caries include the application of pit and fissure sealants on susceptible pits and fissures of the tooth, but these forms of treatment are again costly. The disadvantage of fluoride treatment is that ingestion of excess fluoride can cause skeletal and dental fluorosis. The disadvantage of antibacterial mouth rinses is that even the good bacteria in the oral cavity are killed creating open, noncompetitive surfaces for pathogens to repopulate the oral cavity; moreover, long-term use can cause staining of teeth. Previous studies have suggested that probiotics can be effective in selectively inhibiting oral pathogens and modulating the microbial composition of dental plaque thereby reduce the incidence of dental caries [7-9]. The advantage of probiotics as compared to other preventive methods is that they are generally regarded as safe and have additional health benefits like maintaining a healthy gut. The main pathogen involved in the causation of dental caries is Streptococcus mutans [10]. Probiotics are live microorganisms that when ingested in adequate quantities confer a health benefit to the host. Probiotics in general have been associated with immunity and gut health, and attention has mostly focused on the prevention or treatment of gastrointestinal infections [11]. Probiotics are now known to be efficacious in the treatment of various other diseases which include cardiovascular health, diabetes, weight management, cancer, brain health (anxiety and depression, stress), urogenital health, skin diseases, allergies, and oral health [12-16].

The role of probiotics in oral health management (caries, gingivitis, and periodontal management) is increasingly being recognised with the mechanisms of action considered to be antagonistic activity against pathogens, coaggregation and growth inhibition of pathogens, bacteriocin, organic acid, and hydrogen peroxide production, and interaction with oral epithelium resulting in the reduction of the pathogenicity and cariogenic potential of biofilm microorganisms $[17,18]$. Immunity also appears to have a role with both innate and adaptive immune responses involved in combatting oral diseases [19].

Several studies indicate that the consumption of probiotics can inhibit the levels of mutans streptococci in saliva [20-23]. Strains of Lactobacillus casei, L. rhamnosus, L. reuteri, and Bifidobacterium spp. have demonstrated the ability to inhibit cariogenic bacteria and their colonisation and thus prevent dental caries [24]. A previous study with the spore-forming probiotic strain Bacillus coagulans found that it was effective in reducing the mutans streptococci count [25]. Lactic acid bacteria, the lactobacilli spp. in plaque produce lactic acid and other organic acids from the fermentation of sugars (from the diet of the host) which lead to the progression of dental caries. It is also suggested that in caries-free children, lactobacilli have been found to be largely absent [26]. It was thus of interest to measure the $\mathrm{pH}$, lactobacilli, and mutans streptococci levels in both saliva and plaque of cariogenic children. Since the effects of probiotics are strain-specific, the objective of the present study was to assess the anticariogenic potential of Bacillus coagulans Unique IS2 in dental caries. B. coagulans Unique IS2 is a safe, well-characterized, and stable strain [27, 28], with documented efficacy in restoring digestive health [29-33], reduction of bacterial vaginosis [34], and reduction of side effects of liver cirrhosis [35].

The advantage of $B$. coagulans Unique IS2 is that it is a spore-forming probiotic strain and hence very resilient to harsh manufacturing processes which allows it to be incorporated in any dosage form without loss of viability unlike the vegetative lactobacillus and Bifidobacterium spp. which cannot withstand harsh processes and also require refrigeration. As probiotic effects are not only strain-specific but also dependent on dosage and dosage forms, the aim of this study was to evaluate the efficacy of B. coagulans Unique IS2 chewable tablets on cariogenic organisms in children prone to dental caries.

\section{Methods}

2.1. Study Design. This double-blind, randomized, placebocontrolled study was conducted at the Department of Pedodontics and Preventive Dentistry, SVS Institute of Dental Sciences, Mahabubnagar, Telangana, India.

The study was conducted in accordance with the National Ethical Guidelines for biomedical and health research involving human participants (Indian Council of Medical Research) and the principles of the Declaration of Helsinki. The Ethical Committee of the SVS Institute of Dental Sciences approved the study before initiation of the trial. The trial was also registered with the Clinical Trial Registry- India (CTRI Reg No. CTRI/2017/07/009072).

2.2. Study Population. 126 children were screened, and based on the inclusion and exclusion criteria, 48 children were selected. The sample size of 24 children per group was based on the confidence level (95\%) and margin of error (1\%). There were no dropouts during the study; therefore, all 48 children completed the study (Figure 1). The mean age of the patients was $8.5 \pm 1.6$ years (range of $5-15$ years). 20 (41.67\%) patients were males and $28(58.33 \%)$ patients were females. The children were divided by randomization (computer-generated random numbers) into two equal groups of 24 each-the probiotic or placebo-treated group. The probiotic group consumed $B$. coagulans Unique IS2 chewable tablets ( 2 billion $\mathrm{cfu}$ ) once daily for a period of fourteen days whereas the placebo group was administered placebo chewable tablets for the same length of time. Both the placebo and $B$. coagulans Unique IS2 chewable tablets were manufactured by Unique Biotech Ltd., Hyderabad, India. The placebo chewable tablets differed from the probiotic tablets in that they lacked the active ingredient, $B$. coagulans Unique IS2; otherwise, they were similar with respect to shape, size, and colour to the probiotic chewable tablets. All subjects in the study were of Indian origin and 


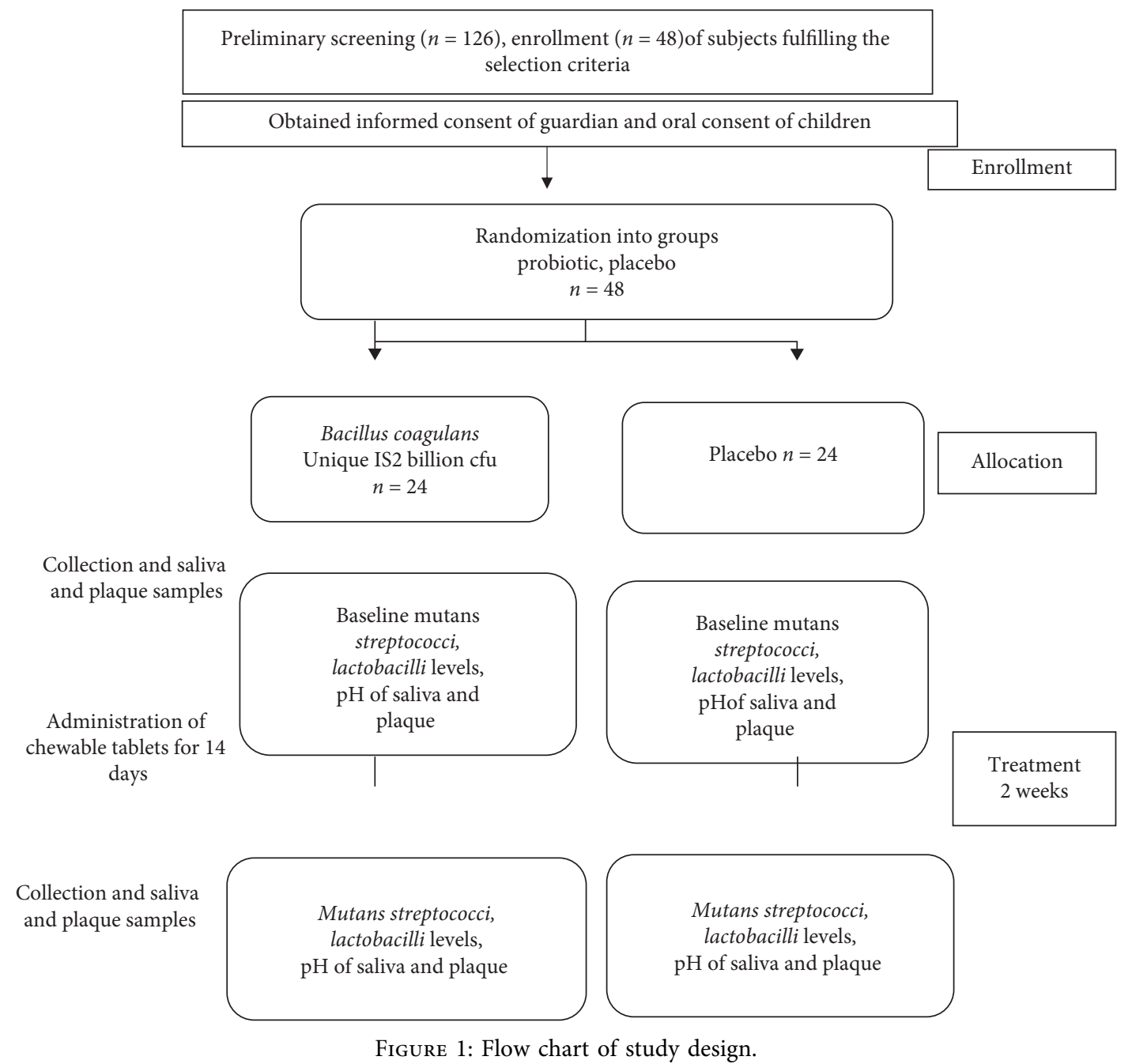

consumed a similar Indian diet of rice, dhal, vegetables, and chapatis (Indian bread). Both the groups were similar with respect to age and sex of the subjects.

Prior to the initiation of the study, children who were selected after screening (on screening day) were provided instructions on oral hygiene and asked to maintain oral health care during the period of treatment. Instructions were provided on the right method of brushing and the need to brush twice daily and rinse vigorously after meals. They were however instructed not to brush their teeth 24 hours prior to Visit 1 (next day after screening and selection) and Visit 2 (after 14 days of treatment) in order to enable plaque collection with ease.

\subsection{Selection Criteria}

2.3.1. Inclusion Criteria. The study included children of either sex between 5 and 15 years of age with increased caries risk (DMFS/defs $\geq 5$ ). Other inclusion criteria: children who had not consumed antibiotics or probiotics in any form three months prior to the study, children who were willing to chew the tablets and participate in the study, and parents of the subjects were willing to give written informed consent and follow study procedures.

2.3.2. Exclusion Criteria. Exclusion criteria included children with severe infections, diseases affecting the whole body, weakened immune systems, and congenital abnormalities; children on fluoride therapy; children using xylitol gum, probiotic products, antibiotics, or corticosteroids within three months of the study initiation; and those not willing to participate in the study.

2.3.3. Randomization. Enrollment of the children who complied with the inclusion/exclusion criteria was done after obtaining signed, written informed consent from the parents. Block randomization was used to divide the children into two treatment arms, the probiotic and placebo-treated groups. Randomization numbers were generated using SAS programming (Statistical Package for Social Sciences, version 18.0, SPSS Inc., Chicago, Illinois, USA). The sealed opaque envelope method was used to keep the study double-blinded. The envelopes with the assignment of probiotic or placebo 
treatment were provided to the clinical site. The investigators in turn disbursed the chewable tablets (probiotic or placebo) to patients based on the randomization numbers.

\subsection{Study Follow-Up Visits and Treatments}

B. coagulans Unique IS2 or placebo chewable tablet was administered once daily for fourteen days. As this was an outpatient study, two visits (Visit 1 and Visit 2) were recorded. Visit 1 was the next day after screening and selection. After collection of saliva and plaque samples for baseline values, treatment was initiated on the same day (Day 1). Visit 2 was the follow-up after the end of treatment (the next day after 14 days of treatment). For both visits, children were instructed not to brush their teeth $24 \mathrm{hrs}$ prior to the visit in order to enable plaque collection with ease.

2.5. Efficacy Parameters. The decrease in the levels of mutans streptococci in saliva and plaque was the primary efficacy parameter studied.

Secondary efficacy parameters assessed were measurement of lactobacilli levels and $\mathrm{pH}$ in both saliva and plaque.

2.6. Collection of Saliva Samples. Stimulated saliva samples were collected in a graded tube to measure $\mathrm{pH}$, mutans streptococci, and lactobacilli levels according to manufacturer's instructions (CRT, Ivoclar Vivadent AG, Schaan, Liechtenstein). Salivary samples were collected at both visits between 9 a.m. and 10 a.m. to avoid any diurnal variations. Children were asked to rinse their mouth thoroughly with distilled water for one minute and then chew on a piece of paraffin wax provided by the manufacturer for five minutes (CRT, Ivoclar Vivadent AG, Schaan, Liechtenstein). The saliva samples were then collected for analysis.

2.7. Collection of Plaque Samples. Sterile toothpicks were used to collect supragingival plaque from all the teeth. Samples were then transferred to a sterile test tube and diluted in a $1: 10$ ratio for all the estimations.

2.8. Estimation of Salivary and Plaque Mutans Streptococci and Lactobacilli. The counts of salivary and plaque mutans streptococci and lactobacilli were estimated with the chairside test (CRT Bacteria, Ivoclar Vivadent AG, Schaan, Liechtenstein) (Table 1) according to the manufacturer's instructions [36].

After identification and counting of the colonies, conversion to scores was done according to the manufacturer's instructions. Scores ranged from 1 to 4 for both mutans streptococci and lactobacilli as per the number of colony forming units (cfu/ml) (Table 1).

2.9. Evaluation of Salivary and Plaque $\mathrm{pH}$. The $\mathrm{pH}$ of saliva and plaque was measured using the chair-side test strip, details of which are provided in an earlier study [37].
TABle 1: Conversion to scores from $\mathrm{Cfu} / \mathrm{ml}$.

\begin{tabular}{|c|c|c|c|c|}
\hline \multicolumn{5}{|c|}{ Scoring for mutans streptococci } \\
\hline $\begin{array}{l}\text { Score } \\
\mathrm{Cfu} / \mathrm{ml}\end{array}$ & $\begin{array}{c}1 \\
<10^{3}\end{array}$ & $\begin{array}{c}2 \\
10^{3}<10^{5}\end{array}$ & $\begin{array}{c}3 \\
10^{5}-10^{6}\end{array}$ & $\begin{array}{c}4 \\
>10^{6}\end{array}$ \\
\hline $\begin{array}{l}\text { Scoring } \\
\mathrm{Cfu} / \mathrm{ml}\end{array}$ & $\begin{array}{l}\text { tobaci } \\
\leq 10^{3}\end{array}$ & $10^{4}$ & $10^{5}$ & $\geq 10^{6}$ \\
\hline
\end{tabular}

2.10. Statistical Analysis. Paired $t$-test was used to compare baseline values to the end of treatment values. The two groups were compared for changes from baseline values using one sample $t$-test and 95\% CI. Measurement data was expressed as means with SD. Values with $P<0.05$ were considered significant.

\section{Results}

\subsection{Primary Efficacy Parameters}

3.1.1. Salivary and Plaque Mutans Streptococci Levels. There was a significant reduction $(P$ value $<0.001)$ in the salivary mutans streptococci levels (as indicated by the scores) in the probiotic treated group after 14 days as compared to the baseline $(3.86 \pm 0.06$ to $2.56 \pm 0.77)$ whereas there was no significant change in the placebo group $(3.08 \pm 0.61$ to $3.18 \pm 0.54)$ (Figure 2 , Table 2$)$. Similarly, in the plaque samples, there was a significant reduction $(P$ value $<0.05$ ) in the mutans streptococci levels in the probiotic treated group after 14 days as compared to the baseline $(2.33 \pm 0.92$ to $1.82 \pm 0.78)$ whereas there was no significant change in the placebo group $(2.71 \pm 0.81$ to $2.56 \pm 0.74)$ (Figure 3, Table 3).

3.1.2. Salivary and Plaque Lactobacilli Scores. There was a significant reduction $(P$ value $<0.001)$ in the salivary lactobacilli levels (as indicated by the scores) in the probiotic treated group after 14 days as compared to the baseline $(3.70 \pm 0.2$ to $2.94 \pm 0.64)$. On the contrary, in the placebotreated group, a significant increase in the lactobacilli levels $(3.33 \pm 0.47$ to $3.83 \pm 0.14)$ was observed (Figure 4 , Table 4$)$. In the plaque samples, the same trend was observed with a significant decrease in lactobacilli levels in the probiotic treated group $(2.71 \pm 0.81$ to $1.54 \pm 0.72)$ and a significant increase in the lactobacilli levels in the placebo group $(2.71 \pm 0.86$ to $3.50 \pm 0.35)$ (Figure 5 , Table 5 ).

3.1.3. pH of Saliva and Plaque Samples. There was no change in the $\mathrm{pH}$ of the saliva samples either with probiotic or placebo treatment with $\mathrm{pH}$ remaining in the range of 7.6-7.8 $(P=0.553)$. Similarly, there was no change in the $\mathrm{pH}$ of the plaque samples with either treatment with $\mathrm{pH}$ in the range of 6.0-6.3 ( $P=0.5030$; data not shown).

\section{Discussion}

The ability of chewable tablets containing the probiotic $B$. coagulans Unique IS2 in lowering the levels of microbes involved in the causation of dental caries, mutans streptococci, and lactobacilli was evaluated in this double-blind, placebo- 


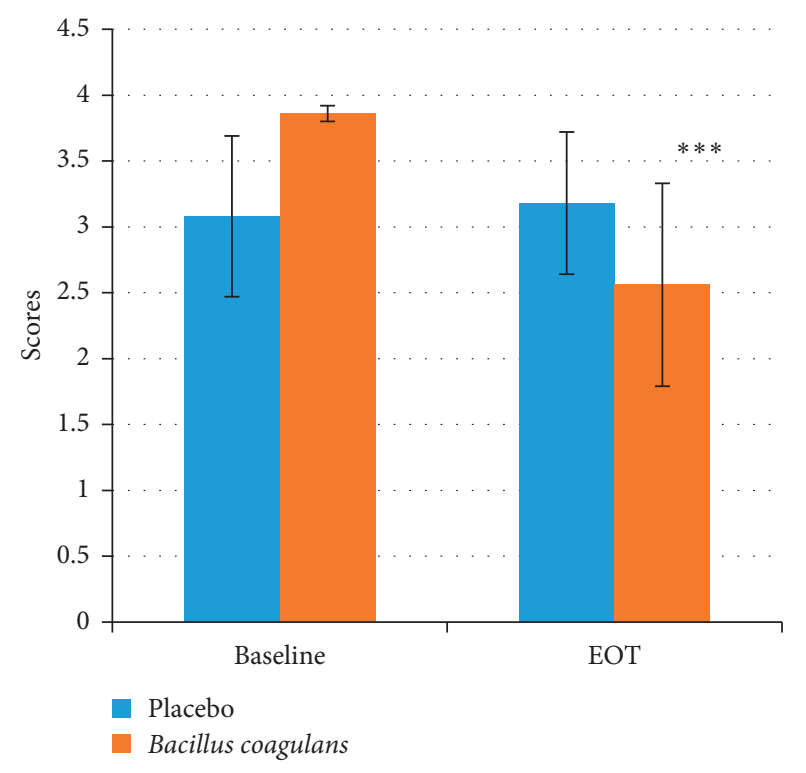

FIGURE 2: Salivary mutans streptococci scores at baseline and EOT. Values are mean \pm s.d. ${ }^{* * *} p$ value $<0.001$.

TABLE 2: Salivary mutans streptococci scores at baseline and EOT.

\begin{tabular}{lccc}
\hline & & Baseline & $\begin{array}{c}\text { End of } \\
\text { treatment }\end{array}$ \\
\hline \multirow{4}{*}{ Placebo } & Min value & 2.39 & 2.39 \\
& Max value & 3.78 & 3.89 \\
& Median & 3.54 & 3.70 \\
& Mean & 3.08 & 3.18 \\
& s.d. & 0.61 & 0.54 \\
\hline \multirow{4}{*}{ Bacillus coagulans Unique IS2 } & Min value & 3.75 & 1.13 \\
& Max value & 3.96 & 3.89 \\
& Median & 3.85 & 2.58 \\
& Mean & 3.86 & 2.56 \\
& s.d. & 0.06 & 0.77 \\
\hline
\end{tabular}

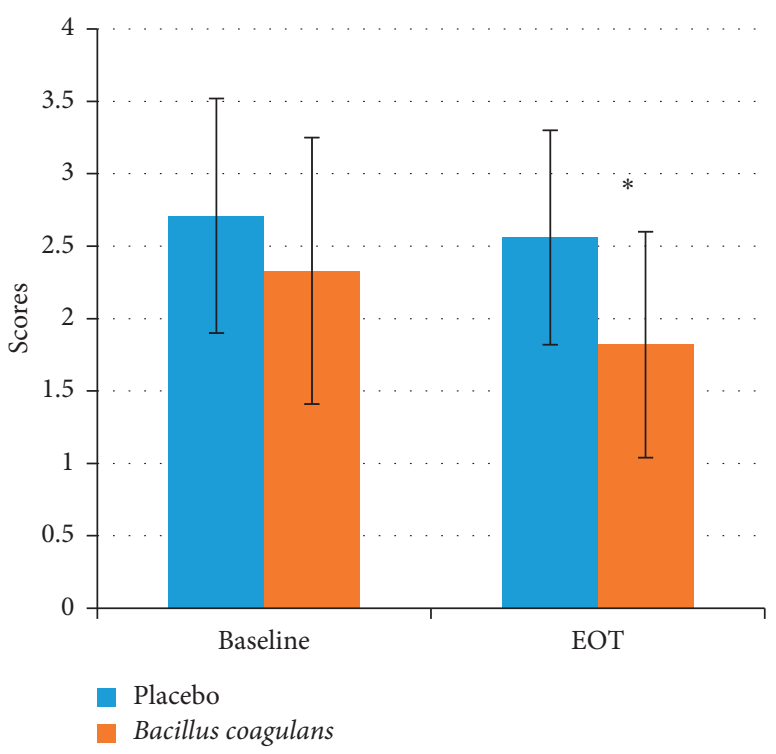

FIgURE 3: Plaque mutans streptococci scores at baseline and EOT. Values are mean \pm s.d. ${ }^{*} p$ value $<0.05$.
TABLE 3: Plaque mutans streptococci scores at baseline and EOT.

\begin{tabular}{lccc}
\hline & & Baseline & $\begin{array}{c}\text { End of } \\
\text { treatment }\end{array}$ \\
\hline \multirow{4}{*}{ Placebo } & Min value & 1.84 & 1.72 \\
& Max value & 3.58 & 3.4 \\
& Median & 1.87 & 1.72 \\
& Mean & 2.71 & 2.56 \\
& s.d. & 0.81 & 0.74 \\
\hline \multirow{4}{*}{ Bacillus coagulans Unique IS2 } & Min value & 1.35 & 1.03 \\
& Max value & 3.28 & 2.62 \\
& Median & 1.36 & 2.51 \\
& Mean & 2.33 & 1.81 \\
& s.d. & 0.92 & 0.78 \\
\hline
\end{tabular}

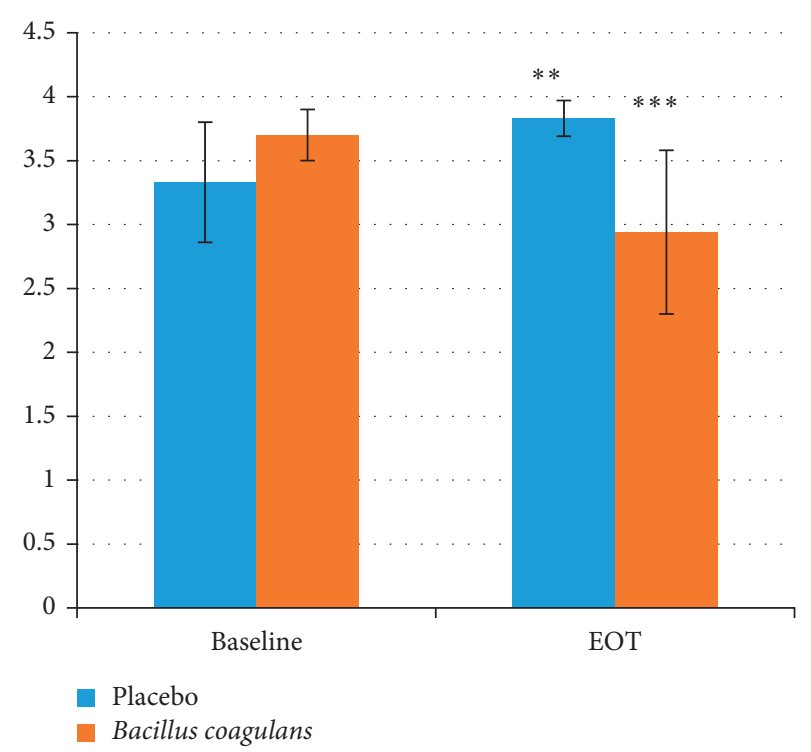

FIgURE 4: Salivary lactobacilli scores at baseline and EOT. Values are mean \pm s.d. ${ }^{* *} p$ value $<0.01 .{ }^{* * *} p$ value $<0.001$.

TABLE 4: Salivary lactobacilli scores at baseline and EOT.

\begin{tabular}{lccc}
\hline & & Baseline & $\begin{array}{c}\text { End of } \\
\text { treatment }\end{array}$ \\
\hline \multirow{4}{*}{ Placebo } & Min value & 2.61 & 3.35 \\
& Max value & 3.9 & 3.92 \\
& Median & 3.61 & 3.88 \\
& Mean & 3.3 & 3.83 \\
& s.d. & 0.47 & 0.14 \\
\hline \multirow{4}{*}{ Bacillus coagulans Unique IS2 } & Min value & 3.28 & 2.24 \\
& Max value & 3.93 & 3.62 \\
& Median & 3.80 & 3.51 \\
& Mean & 3.70 & 2.94 \\
& s.d. & 0.2 & 0.64 \\
\hline
\end{tabular}

controlled study. The subjects included in the study had a propensity towards dental caries (DMFS/defs $\geq 5$ ). It is suggested that the prevalence of mutans streptococci and lactobacilli is increased in plaque and saliva in children with increased caries activity and probiotics can play a role in 


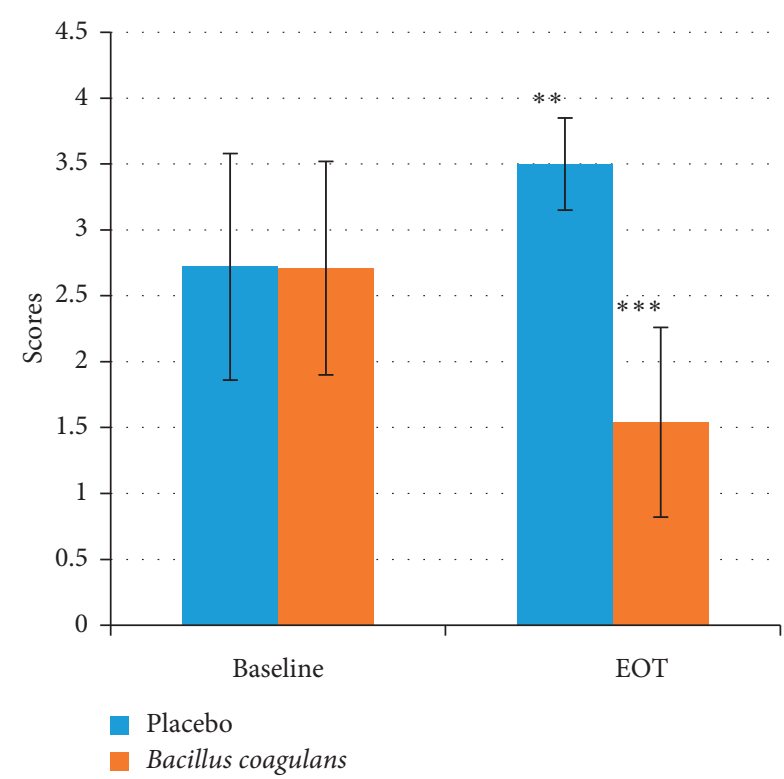

FIGURE 5: Plaque lactobacillus scores at baseline and EOT. Values are mean \pm s.d. ${ }^{* *} p$ value $<0.01 .{ }^{* * *} p$ value $<0.001$.

TABle 5: Plaque lactobacillus scores at baseline and EOT.

\begin{tabular}{lccc}
\hline & & Baseline & $\begin{array}{c}\text { End of } \\
\text { treatment }\end{array}$ \\
\hline \multirow{4}{*}{ Placebo } & Min value & 1.84 & 2.72 \\
& Max value & 3.57 & 3.82 \\
& Median & 3.55 & 3.65 \\
& Mean & 2.72 & 3.50 \\
& s.d. & 0.86 & 0.35 \\
\hline \multirow{4}{*}{ Bacillus coagulans Unique IS2 } & Min value & 1.89 & 0.81 \\
& Max value & 3.52 & 2.26 \\
& Median & 3.5 & 2.25 \\
& Mean & 2.71 & 1.54 \\
& s.d. & 0.81 & 0.72 \\
\hline
\end{tabular}

inhibiting the cariogenic bacteria. Probiotics can therefore be considered as an important alternative therapy for the replacement of pathogenic microorganisms [38-40].

Chewable tablet was chosen as the dosage form for the probiotic supplement as it allows for the probiotic strain to remain longer in the mouth and populates the oral cavity while chewing. The results indicated that daily consumption of $B$. coagulans Unique IS2 probiotic chewable tablets for two weeks effectively reduced the mutans streptococci and lactobacilli counts in both saliva and plaque samples. The duration of two weeks of treatment was chosen based on earlier studies where the efficacy of probiotic strains was demonstrated with the same timeline [25, 41, 42]. This decrease in the levels of mutans streptococci is similar to the findings of some studies $[25,43,44]$. To the best of our knowledge, the efficacy of $B$. coagulans on the levels of salivary or plaque lactobacilli has not been measured. Our study has reported that there is a significant decrease in both the salivary and plaque lactobacilli with $B$. coagulans
Unique IS2 treatment; however, there was no change in the $\mathrm{pH}$ of saliva and plaque samples with either probiotic or placebo treatment. With the significant decrease of lactobacilli spp. in the saliva and plaque samples of the $B$. coagulans Unique IS2 treated group, one would expect an increase in the $\mathrm{pH}$, but this was not observed and could be attributed to the buffering capacity of saliva. A similar observation was made by Villavicencio et al. [40] wherein there was a significant decrease in the lactobacilli spp. in the saliva of children on probiotic treatment but no significant change of $\mathrm{pH}$.

The present report evaluated a selected population of patients, that is, children prone to dental caries. The microbiology of the oral environment can however be altered in other populations by other variables such as restorative frameworks [45] and orthodontic appliances [46]. Additionally, wear can alter surface characteristics of enamel [47] and composite materials [48] and this could have an important influence on bacterial colonisation. Therefore, further studies are needed in order to gain an insight into the role of Bacillus coagulans Unique IS2 in altering the oral microflora in varying oral health conditions. It is known that the genetic make-up of saliva may have an influence on the mutans streptococci levels. Differences in susceptibility to dental caries occur even under the similar, controlled conditions because of genetic variations. Some environmental factors are potentially more cariogenic for some individuals than for others [49]. Most genetic studies have focused on detecting a genetic factor contributing to caries by testing genetic variation, such as single-nucleotide polymorphisms (SNPs) in specific genes, for an association between variants at a genetic locus and caries. These genes are grouped into categories based on the factor influencing dental caries. The major candidate gene categories include enamel formation genes, immune response genes, genes related to saliva, and genes related to taste and dietary habits [50].

B. coagulans Unique IS2 has previously been found to be efficacious in the treatment of diarrhoea [29], constipation [30], and irritable bowel syndrome (IBS) in both adults and children $[31,32]$. In the study with IBS in children, the dosage from $B$. coagulans Unique IS2 (2 billion cfu) was in the form of chewable tablets, the same as used for the present study.

In conclusion, a fourteen-day administration of probiotic B. coagulans Unique IS2 in the form of chewable tablets is able to reduce cariogenic bacteria. A reduction of salivary and plaque mutans streptococci and lactobacilli concentration in children is an indication of reduced caries risk. The limitation of the present study is that it was done with a small population size. Studies with a larger sample size and a longer follow-up are however needed to make any statistically conclusive assertions.

\section{Data Availability}

The data used to support this study were obtained from the Department of Pedodontics and Preventive Dentistry, SVS Institute of Dental Sciences, Mahbubnagar, Telangana, India. 


\section{Conflicts of Interest}

Two of the authors, RS and JN, belong to Unique Biotech Limited which manufactures probiotics including Bacillus coagulans Unique IS2. They state that the study was conducted independently at the Dental Institute with no intervention from them.

\section{Acknowledgments}

Unique Biotech Limited funded the project.

\section{References}

[1] R. G. Watt, B. Daly, P. Allison et al., "Ending the neglect of global oral health: time for radical action," The Lancet, vol. 394, no. 10194, pp. 261-272, 2019.

[2] N. J. Kassebaum, A. G. Smith, E. Bernabé et al., "Oral Health Collaborators. Global, regional, and national prevalence, incidence, and disability-adjusted life years for oral conditions for 195 countries, 1990-2015: a systematic analysis for the global burden of diseases, injuries, and risk factors," Journal of Dental Research, vol. 96, no. 4, pp. 380-387, 2015.

[3] R. Kadel, "Global, regional, and national incidence, prevalence, and years lived with disability for 355 diseases and injuries for 195 countries, 1990-2017: a systematic analysis for the Global Burden of Disease Study 2017," The Lancet, vol. 392, pp. 1789-1858, 2018.

[4] A. Hiremath, V. Murugaboopathy, A. V. Ankola, M. Hebbal, S. Mohandoss, and P. Pastay, "Prevalence of dental caries among primary school children of India-a cross-sectional study," Journal of Clinical and Diagnostic Research, vol. 10, no. 10, p. ZC47, 2016.

[5] R. A. Bagramian, F. Garcia-Godoy, and A. R. Volpe, "The global increase in dental caries. a pending public health crisis," American Journal of Dentistry, vol. 22, no. 1, pp. 3-8, 2009.

[6] N. P. T. Innes, C. H. Chu, M. Fontana et al., "A century of change towards prevention and minimal intervention in cariology," Journal of Dental Research, vol. 98, no. 6, pp. 611-617, 2019.

[7] F. Chen and D. Wang, "Novel technologies for the prevention and treatment of dental caries: a patent survey," Expert Opinion on Therapeutic Patents, vol. 20, no. 5, pp. 681-694, 2010.

[8] X. He, R. Lux, H. K. Kuramitsu, M. H. Anderson, and W. Shi, "Achieving probiotic effects via modulating oral microbial ecology," Advances in Dental Research, vol. 21, no. 1, pp. 53-56, 2009.

[9] P. D. Marsh, "In sickness and in health-what does the oral microbiome mean to us? An ecological perspective," Advances in Dental Research, vol. 29, no. 1, pp. 60-65, 2018.

[10] W. J. Loesche, "Role of Streptococcus mutans in human dental decay," Microbiological Reviews, vol. 50, no. 4, p. 353, 1986.

[11] A. C. Ouwehand, "The role of probiotics in digestive health," Nutrition and Dietary Supplements, vol. 7, pp. 103-109, 2015.

[12] M. Rondanelli, M. A. Faliva, S. Perna, A. Giacosa, G. Peroni, and A. M. Castellazzi, "Using probiotics in clinical practice: where are we now? A review of existing meta-analyses," Gut Microbes, vol. 8, no. 6, pp. 521-543, 2017.

[13] M. R. Sudha, J. J. Ahire, N. Jayanthi, A. Tripathi, and S. Nanal, "Effect of multi-strain probiotic (UB0316) in weight management in overweight/obese adults: a 12-week double blind, randomised, placebo-controlled study," Beneficial Microbes, vol. 10, no. 8, pp. 855-866, 2019.
[14] A.-Q. Yu and L. Li, "The potential role of probiotics in cancer prevention and treatment," Nutrition and Cancer, vol. 68, no. 4, pp. 535-544, 2016.

[15] T. G. Dinan and J. F. Cryan, "The microbiome-gut-brain axis in health and disease," Gastroenterology Clinics of North America, vol. 46, no. 1, pp. 77-89, 2017.

[16] R. P. Allaker and A. S. Stephen, "Use of probiotics and oral health," Current Oral Health Reports, vol. 4, no. 4, pp. 309-318, 2017.

[17] S. Mahasneh and A. Mahasneh, "Probiotics: a promising role in dental health," Dentistry Journal, vol. 5, no. 4, p. 26, 2017.

[18] D. Gruner, S. Paris, and F. Schwendicke, "Probiotics for managing caries and periodontitis: systematic review and meta-analysis," Journal of Dentistry, vol. 48, pp. 16-25, 2016.

[19] G. P. Schincaglia, B. Y. Hong, A. Rosania et al., "Clinical, immune, and microbiome traits of gingivitis and peri-implant mucositis," Journal of Dental Research, vol. 96, no. 1, pp. 47-55, 2017.

[20] A. Haukioja, "Probiotics and oral health," European Journal of Dentistry, vol. 4, no. 3, pp. 348-355, 2010.

[21] E. Çaglar, S. Kavaloglu Cildir, S. Ergeneli, N. Sandalli, and S. Twetman, "Salivary mutans streptococci and lactobacilli levels after ingestion of the probiotic bacteriumLactobacillus reuteriATCC 55730 by straws or tablets," Acta Odontologica Scandinavica, vol. 64, no. 5, pp. 314-318, 2006.

[22] D. Cogulu, A. Topaloglu-Ak, E. Caglar et al., "Potential effects of a multistrain probiotic-kefir on salivary streptococcus mutans and lactobacillus spp," Journal of Dental Sciences, vol. 5, no. 3, pp. 144-149, 2010.

[23] T.-H. Lin, C.-H. Lin, and T.-M. Pan, "The implication of probiotics in the prevention of dental caries," Applied Microbiology and Biotechnology, vol. 102, no. 2, pp. 577-586, 2018.

[24] J. Meurman and I. Stamatova, "Probiotics: contributions to oral health,” Oral Diseases, vol. 13, no. 5, pp. 443-451, 2007.

[25] G. Jindal, R. K. Pandey, J. Agarwal, and M. Singh, "A comparative evaluation of probiotics on salivary mutans streptococci counts in Indian children," European Archives of Paediatric Dentistry, vol. 12, no. 4, pp. 211-215, 2011.

[26] P. W. Caufield, C. N. Schön, P. Saraithong, Y. Li, and S. Argimón, "Oral lactobacilli and dental caries," Journal of Dental Research, vol. 94, no. 9_suppl, pp. 110S-118S, 2015.

[27] R. M. Sudha, M. Sunita, and B. M. Sekhar, "Safety studies of bacillus coagulans unique IS-2 in rats: morphological, biochemical and clinical evaluations," International Journal of Probiotics \& Prebiotics, vol. 11, no. 1, p. 43, 2016.

[28] A. Upadrasta, S. Pitta, and R. S. Madempudi, "Draft genome sequence of the spore-forming probiotic strain Bacillus coagulans unique IS-2," Genome Announcements, vol. 4, no. 2, 2016.

[29] R. M. Sudha and S. Bhonagiri, "Efficacy of Bacillus coagulans strain unique IS-2 in the treatment of patients with acute diarrhea," International Journal of Probiotics \& Prebiotics, vol. 7, no. 1, 2012.

[30] R. S. Madempudi, J. Neelamraju, J. J. Ahire, S. K. Gupta, and V. K. Shukla, "Bacillus coagulans unique IS2 in constipation: a double-blind, placebo-controlled study," Probiotics and Antimicrobial Proteins, vol. 12, no. 2, pp. 335-342, 2020.

[31] M. R. Sudha, N. Jayanthi, M. Aasin, R. D. Dhanashri, and T. Anirudh, "Efficacy of Bacillus coagulans unique IS2 in treatment of irritable bowel syndrome in children: a double blind, randomised placebo controlled study," Beneficial Microbes, vol. 9, no. 4, pp. 563-572, 2018. 
[32] R. S. Madempudi, J. J. Ahire, J. Neelamraju, A. Tripathi, and S. Nanal, "Randomized clinical trial: the effect of probiotic Bacillus coagulans unique IS2 vs. placebo on the symptoms management of irritable bowel syndrome in adults," Scientific Reports, vol. 9, no. 1, pp. 1-9, 2019.

[33] H. Saneian, Z. Pourmoghaddas, H. Roohafza, and A. Gholamrezaei, "Synbiotic containing bacillus coagulans and fructo-oligosaccharides for functional abdominal pain in children," Gastroenterology and Hepatology from Bed to Bench, vol. 8, no. 1, p. 56, 2015.

[34] M. R. Sudha, K. A. Yelikar, and S. Deshpande, "Clinical study of Bacillus coagulans Unique IS-2 (ATCC PTA-11748) in the treatment of patients with bacterial vaginosis," Indian Journal of Microbiology, vol. 52, no. 3, pp. 396-399, 2012.

[35] R. R. Pawar, M. L. Pardeshi, B. B. Ghongane, R. Pawar, M. Pardeshi, and B. Ghongane, "Study of effects of probiotic lactobacilli in preventing major complications in patients of liver cirrhosis," International Journal of Research in Pharmaceutical and Biomedical Sciences, vol. 3, no. 1, pp. 206-211, 2012.

[36] S. K. Cildir, D. Germec, N. Sandalli et al., "Reduction of salivary mutans streptococci in orthodontic patients during daily consumption of yoghurt containing probiotic bacteria," The European Journal of Orthodontics, vol. 31, no. 4, pp. 407-411, 2009.

[37] V. Lakshmi Bolla, S. R. Munnangi, U. K. Chowdary, P. Koppulu, and L. A. Swapna, "Correlation between the $\mathrm{pH}$ of saliva, plaque and buffering capacity of saliva," International Journal of Applied Dental Sciences, vol. 53, pp. 48-50, 2017.

[38] M. Bustamante, B. D. Oomah, M. Rubilar, and C. BurgosDíaz, "Probiotics as an adjunct therapy for the treatment of halitosis, dental caries and periodontitis," Probiotics and Antimicrobial Proteins, vol. 63, pp. 1-10, 2019.

[39] P. Ramanujam, S. Poorni, M. R. Srinivasan, and N. M. Sureshbabu, "Probiotics in dental caries prevention," The Indian Journal of Nutrition and Dietetics, vol. 56, no. 1, p. 84, 2019.

[40] J. Villavicencio, L. M. Villegas, M. C. Arango, S. Arias, and F. Triana, "Effects of a food enriched with probiotics on Streptococcus mutans and Lactobacillus spp. salivary counts in preschool children: a cluster randomized trial," Journal of Applied Oral Science, vol. 26, 2018.

[41] L.-C. Chuang, C.-S. Huang, L.-W. Ou-Yang, and S.-Y. Lin, "Probiotic Lactobacillus paracasei effect on cariogenic bacterial flora," Clinical Oral Investigations, vol. 15, no. 4, pp. 471-476, 2011.

[42] G. S. Pinto, M. S. Cenci, M. S. Azevedo, M. Epifanio, and M. H. Jones, "Effect of yogurt ContainingBifidobacteriumani malissubsp. lactisDN-173010 probiotic on dental plaque and saliva in orthodontic patients," Caries Research, vol. 48, no. 1, pp. 63-68, 2014.

[43] A. Yousuf, A. Nagaraj, S. Ganta et al., "Comparative evaluation of commercially available freeze dried powdered probiotics on mutans streptococci count: a randomized, double blind, clinical study," Journal of Dentistry (Tehran, Iran), vol. 12, no. 10, p. 729, 2015.

[44] M. Koopaie, M. Fatahzadeh, S. Jahangir, and R. Bakhtiari, "Comparison of the effect of regular and probiotic cake (Bacillus coagulans) on salivary $\mathrm{pH}$ and streptococcus mutans count," Dental and Medical Problems, vol. 56, no. 1, pp. 33-38, 2019.
[45] R. Stencel, J. Kasperski, W. Pakieła et al., "Properties of experimental dental composites containing antibacterial silverreleasing filler," Materials, vol. 11, no. 6, p. 1031, 2018.

[46] M. F. Sfondrini, M. Debiaggi, F. Zara et al., "Influence of lingual bracket position on microbial and periodontal parameters in vivo," Journal of Applied Oral Science, vol. 20, no. 3, pp. 357-361, 2012.

[47] M. Ijbara, K. Wada, M. J. Tabata, J. Wada, G. Inoue, and M. Miyashin, "Enamel microcracks induced by simulated occlusal wear in mature, immature, and deciduous teeth," BioMed Research International, vol. 2018, Article ID 5658393, 18 pages, 2018.

[48] A. Scribante, P. Vallittu, L. V. J. Lassila et al., "Effect of longterm brushing on deflection, maximum load, and wear of stainless steel wires and conventional and spot bonded fiberreinforced composites," International Journal of Molecular Sciences, vol. 20, no. 23, p. 6043, 2019.

[49] A. H. Fahad, R. F. Al-Huwaizi, Y. A. Al-Rubbaey, and A. Alhuwaizi, "Salivary $\mathrm{pH}$, flow rate and Streptococcus mutans count in relation to oral health statusamong colored eyes adolescentsin baghdad/Iraq," Biomedical and Pharmacology Journal, vol. 11, no. 4, pp. 2085-2089, 2018.

[50] A. R. Vieira, A. Modesto, and M. L. Marazita, "Caries: review of human genetics research," Caries Research, vol. 48, no. 5, pp. 491-506, 2014. 